\title{
Impact Factor 2006: 1,055 - die Klinische Pädiatrie im Spannungsfeld zwischen klinischer Forschung und Fortbildung
}

\section{Impact Factor 2006: 1.055 - Klinische Pädiatrie in the Tension Field between Clinical Research and Continuing Education}

U. Göbel ${ }^{1}$

F. C. Sitzmann ${ }^{2}$

Institut

${ }^{1}$ Heinrich-Heine-Universität

Düsseldorf

${ }^{2}$ Universitäts-Kinderklinik,

Homburg/Saar

Bibliografie

DOI 10.1055/s-2007-985400

Klin Pädiatr 2007; 219: 249-250

(c) Georg Thieme Verlag KG

Stuttgart · New York

ISSN 0300-8630

Korrespondenzadresse

Prof. em. Dr. U. Göbel

Heinrich-Heine-Universität

Düsseldorf

Moorenstr. 5

40225 Düsseldorf

Goebel@med.uni-duesseldorf.

de
Regelmäßig veröffentlicht in der Mitte eines Jahres das ISI (Institute for Scientific Information, Philadelphia) den „Journal Impact Factor“, der die gemittelte Zitierungsrate der Artikel einer Zeitschrift aus einem Zeitraum von zwei Jahren im darauf folgenden Jahr darstellt. Hier gilt stets der Stichtag 31.12. des betreffenden Jahres.

Der mit großer Spannung erwartete Wert für die Klinische Pädiatrie für das Jahr 2006 entspricht mit 1,055 ziemlich genau dem Wert des Vorjahres [6]. Für die Herausgeber und den Verlag bedeutet dieses erfreuliche Resultat, dass das Ergebnis von 2005 kein einmaliges Ergebnis darstellt ( $\bullet$ Abb. 1) und sich die vorsichtig getroffenen redaktionellen Veränderungen bewährt haben. Relevanter für jede Zeitschrift ist jedoch das Verhalten der Leser und Autoren: Die Klinische Pädiatrie ist also in 2006 in vergleichbarem Umfang wie in 2005 zitiert worden und hat dadurch ihren 2005 errungenen Platz im Mittelfeld der 74 internationalen Zeitschriften, die vom ISI in der Kategorie „Pediatrics“ gelistet sind, verteidigt.

Von Jahr zu Jahr ist bei der Klinischen Pädiatrie eine leichte, aber stetige Zunahme an Veröffentlichungen zu verzeichnen; ein Ausreißer stellt das Jahr 2005 durch die Publikation eines Supplements mit 14 Beiträgen zur „Therapie von Infektionen in der Kinderonkologie“ [11] dar ( $\bullet$ Tab. 1). Für die Fortentwicklung einer jeden Zeitschrift ist es wichtig, Klarheit darüber zu gewinnen, welche Art von Artikel besonders häufig zitiert wird. Deshalb sind die Publikationen in der Tabelle nach der Häufigkeit ihrer Zitierungen sortiert und die kumulierten Zitierungen pro Jahr ermittelt:

- Ganz deutlich wird, dass die Zahl der noch nicht zitierten Arbeiten in erkennbarer Weise abnimmt, je länger ein Jahrgang zurückliegt. Dies kommt vor allem den Rubriken „Kasuistiken“ und „Visite“ zugute, die seltene Krankheitsbilder oder ungewöhnliche klinische Situationen beschreiben und gerade in der Kinderheilkunde mit der geringen Erkrankungsdichte besonders wichtig sind.

- Die Zeit für die Beurteilung der Zitierhäufigkeit der Veröffentlichungen aus dem Jahrgang 2006 ist noch zu kurz, um eine Interpretation zu erlauben.

- Auch langfristig selten oder gar nicht werden Reviews zitiert, obwohl sie besonders häufig gelesen werden. Hier wirkt sich natürlich das
Supplement mit 14 Übersichtsartikeln nachhaltig aus.

- Beiträge mit vier oder mehr Zitierungen stellen auch für jene aus dem Jahr 2004 eine Minderheit dar, wirken sich aber überaus günstig auf den Impact Factor aus.

Da der Impact Factor eines Journals durch einen sehr einfachen Rechenvorgang ermittelt wird, ist klar ersichtlich, dass durch ihn nicht der wissenschaftliche Wert einer einzelnen Publikation wiedergegeben werden kann. Trotzdem wird dieser Bewertungsmaßstab weithin eingesetzt, u.a. von den Fakultäten zur Evaluation der publikatorischen Leistung besonders von jungen Wissenschaftlern oder auch von ganzen Institutionen bzw. den Wissenschaftsministerien bei der leistungsbezogenen Mittelvergabe für die Forschung. Anstatt den Impact Factor einer Zeitschrift anzugeben, wird gelegentlich vorgeschlagen, die Zahl der Zitierungen für die einzelne Arbeit während eines genügend großen Zeitintervalls heranzuziehen. Da in der Zeitschrift Klinische Pädiatrie knapp die Hälfte der Veröffentlichungen aus den Jahren 2005 und 2006 aus der Allgemeinen Pädiatrie und die andere Hälfte von Mitgliedern der Gesellschaft für Pädiatrische Onkologie und Hämatologie (GPOH) erstellt worden ist, kann die Gegenüberstellung der drei jeweils am häufigsten zitierten Beiträge aus diesen Jahren auf-

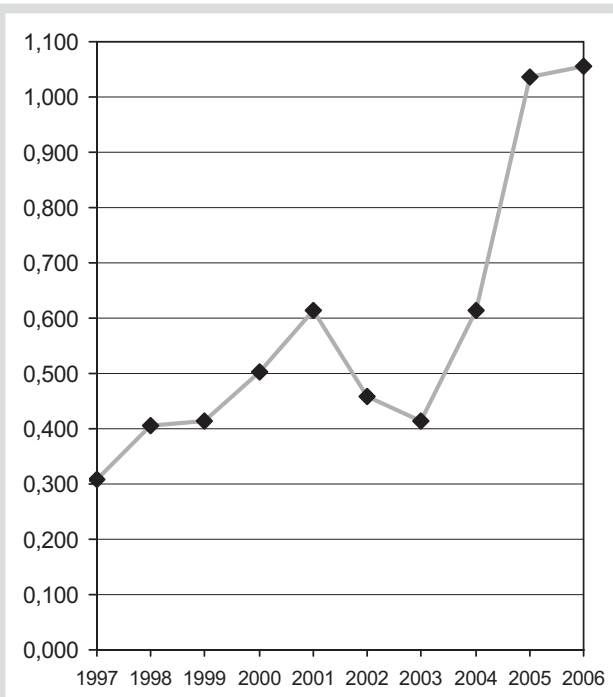

Abb. 1 Der Verlauf der Journal Impact Factors für die Klinische Pädiatrie über 10 Jahre. 
Tab. 1 Zitierung der Arbeiten der Klinischen Pädiatrie aus den Jahren 2006, 2005 und 2004 (Quelle: Science Citation Index Expanded, ISI Web of Knowledge ${ }^{\mathrm{SM}}$; Stichtag: 31.06.2007)

\begin{tabular}{|c|c|c|c|c|c|c|c|}
\hline Jahr & $\begin{array}{l}\text { Anzahl der } \\
\text { Zitierungen }\end{array}$ & 0 & $1-3$ & $4-6$ & $7-9$ & $\geq 10$ & $\Sigma$ \\
\hline \multirow[t]{2}{*}{2006} & $\begin{array}{l}\text { Anzahl der } \\
\text { Arbeiten }\end{array}$ & 53 & 10 & - & - & - & 63 \\
\hline & $\begin{array}{l}\text { kumulierte } \\
\text { Zitate }\end{array}$ & 0 & 12 & - & - & - & 12 \\
\hline \multirow[t]{2}{*}{2005} & $\begin{array}{l}\text { Anzahl der } \\
\text { Arbeiten }\end{array}$ & 45 & 23 & 4 & 2 & - & $74^{*}$ \\
\hline & $\begin{array}{l}\text { kumulierte } \\
\text { Zitate }\end{array}$ & 0 & 45 & 19 & 16 & - & 80 \\
\hline \multirow[t]{2}{*}{2004} & $\begin{array}{l}\text { Anzahl der } \\
\text { Arbeiten }\end{array}$ & 15 & 26 & 7 & 5 & 3 & 56 \\
\hline & $\begin{array}{l}\text { kumulierte } \\
\text { Zitate }\end{array}$ & 0 & 45 & 33 & 36 & 44 & 158 \\
\hline
\end{tabular}

schlussreich sein. Bei den sechs Publikationen aus der Allgemeinen Pädiatrie handelt es sich viermal um klinische Beobachtungsstudien $[2,8,13,15]$, eine randomisierte Prüfung [16] und eine innovative Pilotstudie [12], die alle monoinstitutionell durchgeführt wurden. Dagegen haben zwei der GPOH-Beiträge mit besonders häufiger Zitierung eine gesundheitspolitische Zielrichtung [3,5], drei weitere sind kooperative und interdisziplinär angelegte Projekte $[7,10,14]$ und nur eine Arbeit ist eine monoinstitutionelle Pilotstudie - allerdings mit translationalen Aspekten [9]. Derartige Verfahren bedeuten eine Arzneientwicklung in der Kinderonkologie [1], die die Vorgaben der 12. Novelle des Arzneimittelgesetzes zu berücksichtigen haben [5] und an deren Erfüllung kooperativ gearbeitet wird [4].

Positiv bemerkbar macht sich ein hoher Grad der überregionalen und interdisziplinären Kooperationen, die zu einer höheren Patientendichte führen und entsprechende Strukturen erfordern. Das wirkt sich erheblich auf die Zitierungsrate aus. Diese vorwiegend formale Betrachtung zeigt, dass der Journal Impact Factor in seiner üblichen Anwendung ein brauchbares Instrument ist, obwohl Fachgebiete mit geringer Krankheitsdichte grundsätzlich benachteiligt sind und aktuelle Trends in besonderer Weise unterstützt werden. Grundsätzlich andere Bedingungen bestehen für Zeitschriften, die sich auf Beiträge der Grundlagenforschung ausrichten, die bei Vorliegen von Innovationen besonders häufig zitiert werden.

\section{Literatur}

1 Benninger-Döring G, Boos J. Arzneimittelentwicklung in der Kinderonkologie. Klin Pädiatr 2006; 218: 152-156

2 Borte $M$, Oertelt $C$, Högy B. Behandlung von Patienten mit primären Antikörpermangelsyndromen in Deutschland. Klin Pädiatr 2005; 217: $276-280$

3 Creutzig $U$, Jürgens $H$, Herold R, Göbel $U$, Henze G. Konzepte der GPOH und des Kompetenznetzes zur Weiterentwicklung und Qualitätssicherung in der Pädiatrischen Onkologie. Klin Pädiatr 2005; 216: 379-283

4 Dilloo D, Niemeyer C, Suttorp M, Vormoor J. Dauer der Einlagerung autologer Blutstammzellprodukte von Kindern und Jugendlichen mit malignen Erkrankungen. Klin Pädiatr 2005; 217: 371-373

5 Graf N, Göbel U. Therapieoptimierungsstudien der Gesellschaft für Pädiatrische Onkologie und Hämatologie (GPOH) und 12. Novelle des Arzneimittelgesetzes zur Umsetzung der EU-Richtlinie. Klin Pädiatr 2004; 216: 129-131

6 Herausgeber und Verlag:. In eigener Sache - Impact Factor 2006: 1.036. Klin Pädiatr 2006; 218: 247-248

7 Kaatsch P, Blettner M, Spix C, Jürgens H. Das Langzeit-Follow-up in der deutschen pädiatrischen Onkologie als Basis für die Durchführung von Studien mit Langzeitüberlebenden. Klin Pädiatr 2005; 217: 169-175

8 Klepper J, Leiendecker B, Riemann E, Baumeister FAM. Die ketogene Diät in den deutschsprachigen Ländern im Jahr 2003: eine Standortbestimmung. Klin Pädiatr 2005; 216: 277-287

9 Lang P, Schumm M, Greil J, Bader P, Klingebiel T, Müller I, Feuchtinger $T$, Pfeiffer M, Schlegel PG, Niethammer D, Handgretinger R. Ein Vergleich von Methoden zur Gewinnung hämatopoetischer Stammzellen für die haploidentische Transplantation: Erste Resultate einer Pilotstudie. Klin Pädiatr 2005; 217: 334-338

10 Langer T, Stöhr W, Paulides M, Kremers A, Dörr HG, Göbel U, Beck JD. Prospektive multizentrische Erfassung von schweren Spätfolgen bei Sarkompatienten im Late-Effects-Surveillance-System (LESS). Klin Pädiatr 2005; 217: 176-200

11 Laws H-J, Lehrnbecher Th (Hrsg): Therapie von Infektionen in der Kinderonkologie. Klin Pädiatr 2005; 217: 1-174

12 Müller H, Weiß C, Kuntz S, Akkoyun I, Schaible T. Gibt es Prädiktoren für proliferierende Stadien der Retinopathia praematurorum und ist eine Sauerstoffsupplementation als konservative Therapiemöglichkeit sinnvoll? Klin Pädiatr 2005; 217: 53-60

13 Pape L, Gunzer F, Ziesing S, Pape A, Offner G, Ehrich JHH. Bakterielle Erreger, Resistenzentwicklung und Behandlungsoptionen beim ambulant erworbenen Harnwegsinfekt im Kindesalter. Klin Pädiatr 2005; 216: $83-86$

14 Reinhard H, Semler O, Bürger D, Bode U, Flentje M, Göbel U, Gutjahr P, Leuschner I, Maaß E, Niggli F, Scheel-Walter HG, Stöckle M, Thüroff JW, Tröger J, Weirich A, Schweinitz D von, Zoubek A, Graf N. Ergebnisse der Therapiestudie SIOP 93-01/GPOH für die Behandlung von Patienten mit unilateralem nichtmetastasierendem Wilms-Tumor. Klin Pädiatr 2005; 216: 132-140

15 Reinehr T, Kersting M, Wollenhaupt A, Alexy U, Kling B, Ströbele K, Andler $W$. Evaluation der Schulung „OBELDICKS“ für adipöse Kinder und Jugendliche. Klin Pädiatr 2005; 217: 1-8

16 Yalaz M, Arslanoglu S, Akisu M, Atik T, Ergun O, Kultursay N. Vergleich der Wirkung zweier natürlicher Surfactantpräparate in der Therapie des Atemnotsyndroms Frühgeborene. Klin Pädiatr 2005; 216: 230-237 